\title{
Lag Camera: A Moving Multi-Camera ArRay FOR SCENE ACQUISITION
}

\author{
Daniel G. Aliaga \\ $\mathrm{Yi} \mathrm{Xu}$ \\ Voicu Popescu \\ $\{$ aliaga|xu43|popescu\}@cs.purdue.edu \\ Department of Computer Science at Purdue University \\ West Lafayette, IN - USA
}

\begin{abstract}
Many applications, such as telepresence, virtual reality, and interactive walkthroughs, require a three-dimensional (3D) model of real-world environments. Methods, such as lightfields, geometric reconstruction and computer vision use cameras to acquire visual samples of the environment and construct a model. Unfortunately, obtaining models of real-world locations is a challenging task. In particular, important environments are often actively in use, containing moving objects, such as people entering and leaving the scene. The methods previously listed have difficulty in capturing the color and structure of the environment while in the presence of moving and temporary occluders. We describe a class of cameras called lag cameras. The main concept is to generalize a camera to take samples over space and time. Such a camera, can easily and interactively detect moving objects while continuously moving through the environment. Moreover, since both the lag camera and occluder are moving, the scene behind the occluder is captured by the lag camera even from viewpoints where the occluder lies in between the lag camera and the hidden scene. We demonstrate an implementation of a lag camera, complete with analysis and captured environments.
\end{abstract}

Keywords: computer graphics, space-time stereo, lightfields, lumigraphs, foreground object removal, camera clusters.

\section{INTRODUCTION}

Computer graphics applications such as telepresence, virtual reality, and interactive walkthroughs require a three-dimensional (3D) model of real-world environments. Students can "visit" famous historical sites, such as museums, temples, battlefields, and distant cities; archeologists can capture excavation sites as they evolve over time; soldiers and fire fighters can train in simulated environments; real estate agents can show potential buyers interiors of homes for sale via the Internet; and, people world-wide can enjoy virtual travel and 3D games. Thus, a growing desire exists for methods which can efficiently capture important and visually stunning environments.

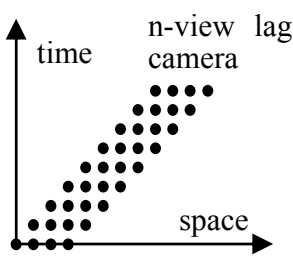

(a)

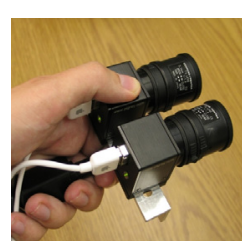

(b)

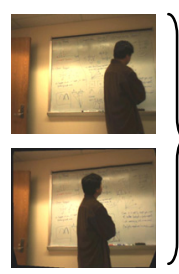

(c)

Numerous methods, such as lightfield acquisition and geometric reconstruction, use cameras to acquire visual samples of the environment and construct a model. Unfortunately, efficiently obtaining models of complex real-world locations is a challenging task. In particular, the aforementioned important environments are often actively in use, containing moving objects, such as people entering and leaving the scene. The methods listed have difficulty in capturing the color and structure of the environment while in the presence of moving and temporary occluders. An efficient acquisition process must be able to proceed even in such situations.

Our key idea is to generalize the concept of a camera to take samples over space and time. Such a camera can easily and interactively detect and sample

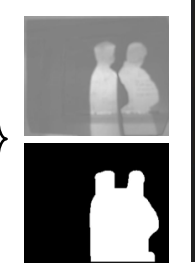

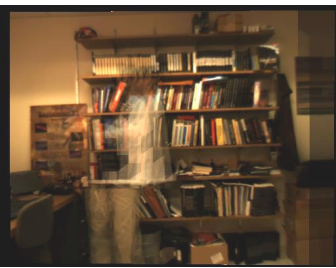

(d)

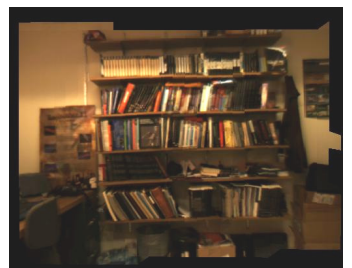

(e)

Figure 1. Lag Camera. (a) The general n-view lag camera samples a scene over space and time by ensuring at least one camera "lags" behind ( $\mathrm{n}=4$ in figure). (b) A snapshot of our prototype 2-view lag camera. (c) Using images acquired from the same viewpoint but at two instances in time, we can easily identify foreground motion as well as acquire samples of the background in the presence of moving occluders. This allows us to capture busy, in-use environment despite temporary occluders appearing and disappearing from the images. Rendering results without (d) and with (e) our method are shown. 
moving objects while continuously moving through the environment. As opposed to the operator having to interrupt capture or having to purposefully avoid occluders, our approach frees the operator completely from having to worry about moving occluders. Moreover, the camera is able to acquire images of the (hidden) scene regardless of the occlusions produced by moving obstacles. This reduces capture time by avoiding having to revisit temporarily occluded surfaces. For example, acquisition can use a hand-held version of our camera design to acquire a lightfield of a large statue even if people are walking in the fieldof-view of the camera and between the camera and the statue (as would often occur for an important structure on display). Our camera system can be mounted on a car and used to acquire images of the architectural structures of an urban neighborhood even in the presence of other moving cars and people. Our camera can be used to simultaneously move within and capture images of a large museum even during normal operation hours when the museum is full of visitors. Since acquisition of a large environment requires significant time, it is unrealistic to ask site supervisors to close-down a location for a lengthy capture session. However, visually stunning, important, and thus actively-used sites are precisely the ones we are interested in capturing.

In this paper, we introduce a class of cameras called lag cameras. The main concept is to have a small handheld cluster of cameras where at least one camera follows (or "lags") behind a lead camera and to interactively acquire space-time samples of the environment. In particular, follow cameras capture the scene from approximately the same viewpoints as lead cameras but at later instances in time. A lag camera supports and assists various space-time processing methods including space-time stereo, foreground object processing, environment reconstruction, and lumigraphs and lightfields. Figure 1a illustrates a space-time sampling of an environment using a general $\mathrm{n}$-view lag camera. The horizontal axis corresponds to samples taken over 3D space. The vertical axis corresponds to samples taken over time. As the cameras move, the lead camera (rightmost camera in Figure 1a) moves to a new viewpoint and all other cameras follow and capture views of the environment from previously visited viewpoints but at later instances in time. Figure $1 \mathrm{~b}$ shows a picture of our first implementation of a lag camera using only two cameras. Figure 1c shows example images captured by our lag camera. These images are acquired from approximately the same viewpoint and at nearby instances in time. Hence, a lag camera can move through the environment, efficiently capturing image samples, yet easily and interactively detecting moving foreground objects. Moreover, since both the occluder and the lag camera are moving, the system can both omit foreground objects and obtain samples of surfaces behind the occluder. Figure 1e shows an example application of lag cameras to produce novel views using a modified unstructured lumigraph. Figure 1d shows a naïve reconstruction from the same viewpoint with a moving occluder partially appearing.

Our main contributions are as follows:

- We describe lag cameras that can move through an environment while acquiring space-time samples of the scene.

- We develop a new motion detection algorithm using a lag camera to create motion masks. This technique allows us to easily and interactively detect moving objects in the scene while the camera itself is undergoing motion. The detected moving objects can then be extracted, reconstructed or removed from the capture.

- We provide a method for acquiring samples of a static background scene even in the presence of moving occluders in between the camera and the scene. This allows us to acquire images of in-use environments even if people enter and leave the field of view of the camera.

\section{RELATED WORK}

Our lag camera design borrows ideas from multiple areas of research. Previous methods have used camera clusters to increase field of view or construct stereo rigs. In contrast, the purpose of a lag camera, while also a cluster of cameras, is to acquire space-time samples for foreground and background sampling.

Space-time sampling addresses the 3D reconstruction problem by sampling in both spatial and temporal domains. For example, Davis et al [Davis 2005] use a spatial-temporal algorithm to reconstruct depth for moving scenes. Similar work can also be found in [Zhang 2003]. Our method is related to these approaches in the sense of supporting such space-time algorithms. Instead of using regular cameras, we propose a novel camera design that is well-suited for space-time processing.

Lightfield and lumigraph rendering methods capture and represent the scene with a group of images [Levoy 1996, Gortler 1996, Buehler 2001]. Rays are selected and blended from the reference images to create novel views of the scene without explicit knowledge of the geometry of the scene. Standard lightfield and lumigraph methods are restricted to static scenes and thus to the space domain. There exist 


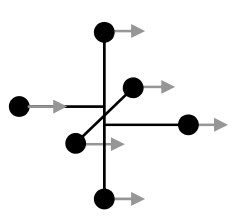

(a)

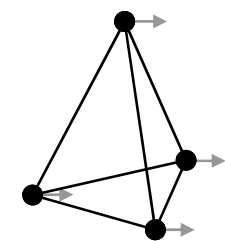

(b)

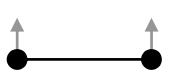

(c)

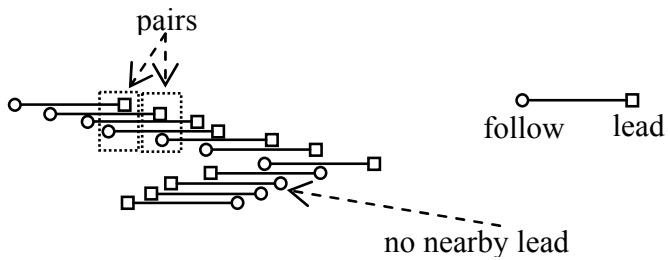

(d)

Figure 2. Lag Camera Configurations. There are many possible lag camera configurations. For instance, (a) a six camera design (each filled dot represents a camera), (b) a four camera design, and (c) a two camera design which provides a follow and lead camera only for camera motion parallel to the baseline. A user simply zigzags this lag camera from one side to another or moves the camera along a smooth continuous path. (d) This figure shows a top view of a capturing path. For each follow camera, the closest lead camera is found.

methods that attempt to capture and render dynamic scenes using lightfield and lumigraph rendering [Naemura 2002, Yang 2002, Matusik 2004]. These methods treat images captured at each time instance as an independent lightfield. Hence, no temporal coherence information is exploited in these methods. Space-time lightfield rendering [Wang 2005a] interpolates the reference views in both temporal and spatial domain using an array of static cameras. After establishing feature correspondence among successive frames for each camera, new images are synthesized in the temporal domain using a novel edge-guided image morphing algorithm. Then these synthesized images are used to interpolate the final rendering result spatially. Our method, on the other hand, acquires spatial and temporal samples using a moving cluster of cameras and with real-time algorithms.

Our method is also related to algorithms for foreground object cutout. Interactive video cutout allows users to cut foreground objects from video sequences [Wang 2005b]. They use a volumetric painting interface to manually select rough approximations of foreground objects and use a computationally-expensive hierarchical segmentation algorithm to refine the selection of foreground objects. Afterwards, a spatial-temporal alpha matting method is used to composite objects onto new background. A similar work is presented in [ $\mathrm{Li} 2005]$. Our method is designed to automatically and interactively detect moving foreground objects for a moving camera - a particularly difficult case.

Finally, our work is also related to video textures [Schödl 2000] and panoramic video textures [Agarwala 2005]. A video texture uses a sampling of a scene over time but from a static viewpoint. The captured images are re-arranged into a seemingly infinite and continuous video of periodic motion in the scene. A panoramic video texture acquires images over time and with a camera rotating in place. Using dynamic programming and a hierarchical min-cut optimization algorithm, the method stitches subsets of the captured imagery together producing an apparent large field-of-view continuous video of periodic motions in the surrounding environment. In contrast, our technique is able to capture more than one temporal sample from each viewpoint as the lag camera moves (in 3D) within the scene. Our spatial and temporal sampling strategy allows us to implement methods for easily detecting moving objects (focusing on either reconstructing the static background or the moving objects), capturing samples of surfaces temporarily occluded, and improving acquisition efficiency by allowing the camera to continuously move and capture in-use environments.

\section{Designing a lag Camera}

\subsection{Camera Configurations}

There are many possible configurations for a general n-view lag camera. A lag camera consists of a compact and handheld cluster of standard cameras. The overall design must take into account the expected type of camera motion and scene motion. In particular, there are four general cases: (1) static camera and static scene, (2) static camera and moving scene, (3) moving camera and static scene, and (4) moving camera and moving scene. A lag camera essentially maps the most difficult case (moving camera and moving scene) to the case of a static camera and a moving scene and enables the use of all algorithms for static cameras. Thus, the design focuses on making a moving camera act like a static camera for at least a small time interval.

Figure 2 demonstrates camera arrangements for several camera motions. Each design must be accurately manufactured or calibrated in software. Figure 2a provides a design for follow cameras that precisely lag behind lead cameras for all motions parallel to either $\mathrm{x}$ - or $\mathrm{y}$-axis and approximately lags behind for motion parallel to the z-axis. Figure $2 \mathrm{~b}$ depicts a configuration with less cameras that provides 
approximate follow and lead cameras for any movement direction. By placing cameras on the corners of a regular tetrahedron, follow cameras will almost trace the path of lead cameras. Figure 2c shows a single-axis lag camera for motion along a single axis. This is the design we implemented for the experiments in this paper. It lets the user move the camera along any straight line in 3D space; but, the user must keep the baseline of the camera-pair approximately parallel to the movement direction. The velocity vector of the lag camera can be used to easily determine the lead camera and when the cameras are not moving in a direction parallel to the baseline. We found this to be a simple and intuitive motion to perform with a portable camera (Figure 2d).

Although Figure 2c illustrates a design using two cameras, the miniaturization of technology makes it easy to extend the configuration to more cameras packed tightly together. In fact, a shorter baseline between cameras of the lag camera cluster and the higher frame rates of future technology actually improves the performance of the lag camera by enabling a larger ratio between object velocity and camera velocity. Nevertheless, the system would have to store more images and support higher bandwidths to maintain interactivity.

\subsection{Lag Camera Image PaIRS}

Given a lag camera configuration, we attempt to pair a follow camera image with the closest and previously obtained lead camera image from ideally the same viewpoint. The lead camera image paired to a follow camera image for this purpose is not necessarily from exactly one previous frame-time ago nor do their viewpoints necessarily coincide (Figure 2d, Figure 3). Rather, the pairing of lead camera and follow camera images depends on the camera motion. Moreover, because of discrete frame rates, the optical axis of the cameras not being exactly parallel to each other, and the freedom of handheld motions, the follow camera will not fall exactly on the viewpoint and view direction of the lead camera (in practice, it is off by only a few millimeters).

To address the aforementioned problems, we warp the lead camera image to the follow camera viewpoint. As a preprocess, we obtain the internal and relative external parameters of each camera using calibration techniques. Then, using a simple proxy of the environment (e.g., we use simples planes to represent the ground and walls in all our scenes), we warp the lead camera image to the follow camera viewpoint. We do not require dense depth information or have to compute depth on the fly. Rather, the proxy provides

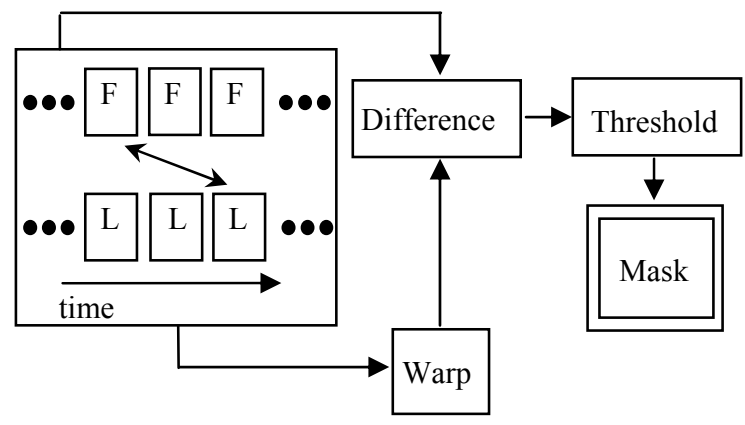

Figure 3. Computing Motion Masks. Each follow frame $\mathrm{F}$ is paired with a lead frame $\mathrm{L}$. $\mathrm{L}$ is warped to the viewpoint of $\mathrm{F}$. A difference image of frame $\mathrm{F}$ and Warp $(\mathrm{L})$ is computed. The motion mask is generated by thresholding the difference image.

very approximate information but that is sufficient to align the images in the pair. They are typically handmade and consist of a few planes or a box. To compensate for remaining inaccuracies, we further align the two images by morphing one image to the other using a sparse set of automatically tracked and corresponded point features between the two images.

\section{Foreground OBJECTS}

\subsection{Motion MASKS}

As the lag camera moves through the environment, it is able to detect (sample) foreground motion by simply comparing a follow camera image to its paired lead camera image. Then, a moving object can be explicitly captured or intentionally removed from the images. The simplest method for identifying object motion in captured imagery is to create a motion mask (Figure 3 ). Although we could produce different masks for each camera, for simplicity we produce a single mask for both images. The mask indicates which subset of the lead camera and follow camera image can be used, for instance, in lumigraph rendering, feature tracking, or $3 \mathrm{D}$ reconstruction.

To create the mask, the system generates on-the-fly thresholded difference images. After warping the lead camera image to the follow camera viewpoint, the images are smoothed using a Gaussian blur and subtracted from each other to produce a difference image. The absolute value difference image is thresholded to produce a binary image which is then subject to an image processing "close" operator to join nearby image components. The contours of all blobs are found and filled (small contours are considered noise and removed). Since warping the lead camera image to the follow camera viewpoint does not 
produce exactly overlapping images, a thin image border is excluded during processing.

Objects which temporarily stop in the scene can still be detected and an appropriate object mask created. When a moving object stops, its difference image becomes zero. However, a motion exists before (and after) the stop. Thus, a sudden disappearance (or appearance) of a contour signals a temporarily stationary object. We can re-project the masks of the frames immediately preceding and succeeding the stop frames to the frame where the difference image goes to zero. Once an object stops for too long, we can optionally choose to call it background.

Multiple objects in the scene can also move and/or stop. To support this situation, we compose masks together. A mask is composed of a combination of masks for moving objects and re-projections of masks for temporarily stationary objects. Figures 7 and 8 (in the results section) demonstrate this functionality.

\subsection{OBJECT DiSPLACEMENT}

Given a lag camera design, we can use rough estimates of typical object distances and velocities (e.g., assume the camera is handheld, assume the scene contains walking humans, etc) to better understand the motion masks and to estimate the sensitivity of the lag camera to the motions. More precisely, the image-space displacement of an object from a follow camera image

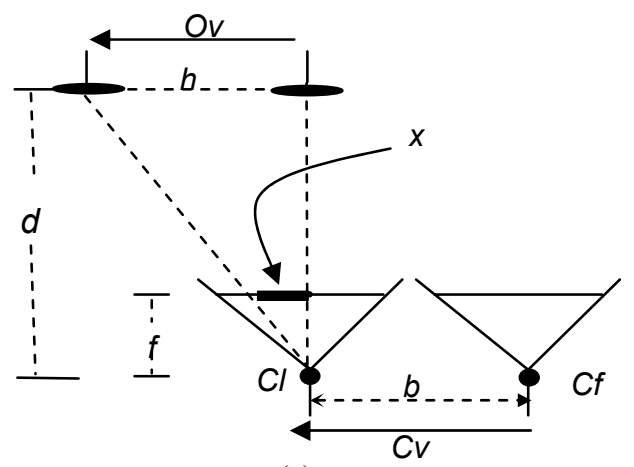

(a)

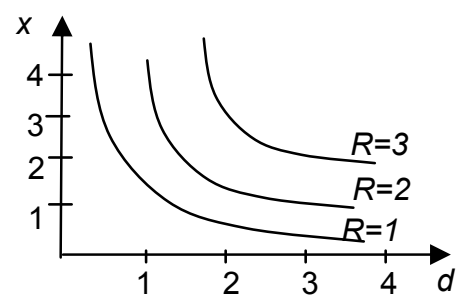

(b)

Figure 4. Object Displacement. (a) Given the baseline b, focal length $f$, depth of the scene $d$, the camera velocity $c_{v}$ and object velocity $o_{v}$, we can estimate the object displacement $\mathrm{x}$ on the two frames. (b) graphs $\mathrm{x}$ as a function of $\mathrm{d}$ for various $\mathrm{R}$ values.

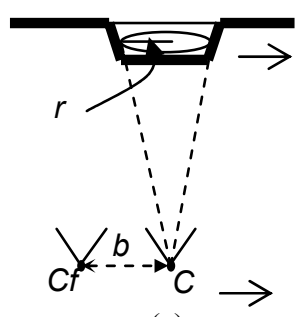

(a)

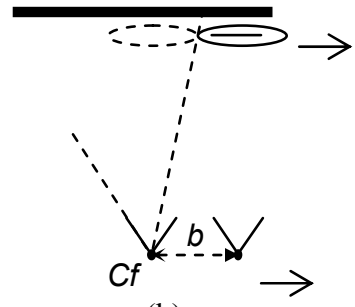

(b)

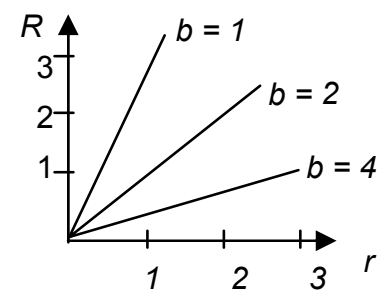

(c)

Figure 5. Background Sampling. (a) Part of the surface is occluded by the object. Camera and the object are moving to the right. (b) Follow camera is now at same location as previous lead camera. Occluded surface can be seen as long as the object does not overlap in volume. (c) Graphs the minimum value of R to achieve full sampling of background.

to its warped lead camera image causes a double image of the moving object to appear in the difference image. The double image may either partially overlap or be completely disjoint. In either case, the displacement between object centers is called $x$. The displacement direction corresponds to the projection of the object velocity onto the image plane of the lag camera.

Figure $4 \mathrm{a}$ contains a diagram of a lag-camera viewing a moving object. The lead camera $c_{l}$ is separated from the follow camera $c_{f}$ by a baseline $b$. The velocity of both cameras is $c_{v}$. The object $o$ displaces significantly when it is moving in a direction parallel to $c_{v}$, such as $o_{v}$. (In this figure both $c_{v}$ and $o_{v}$ are in the same direction. The discussion that follows works equally if $c_{v}$ and $o_{v}$ are moving in opposite directions.) The distance from the cameras to the objects is $d$ and the focal length of the cameras is $f$. Thus, the time it takes the follow camera to reach the position of the lead camera is $t_{c}=b / c_{v}$. In this amount of time, the object has moved by $h=o_{v} t_{c}$ in world space. If we define the ratio of the object velocity $o_{v}$ to the camera velocity $c_{v}$ to be $R$ and use similar triangles, this brings us to the following simplified expression for the magnitude of the screen-space displacement $x$ of the object: $|\mathrm{x}|=|(\mathrm{fb} / \mathrm{d}) \mathrm{R}|$.

This expression is useful to estimate how much the object will move from follow camera image to the corresponding warped lead camera image. For a given lag camera, we can consider $f$ and $b$ to be constants. 
Table 1. Captured Scenes. We show the names, description, and number of images for our example sequences.

\begin{tabular}{|l|l|l|}
\hline Scene & Description & No. Images \\
\hline walk & Student walks into scene and walks out. & 142 \\
\hline walk-stop-walk & Student walks into scene, stops to read the board, and walks out. & 102 \\
\hline walk-hi-walk & Student walks into scene, says hi, waves, and walks out. & 126 \\
\hline walk-head-walk & $\begin{array}{l}\text { Student walks into scene, appears surprised, and another guy appears, both } \\
\text { walk away. }\end{array}$ & 146 \\
\hline motion in office & Student tries his best to hide the background in an office scene during capture. & 298 \\
\hline
\end{tabular}

Figure $4 \mathrm{~b}$ shows a graph of $x$ as a function of $d$ for several values of $R$. In this graph, we use the focal length $f(.377 \mathrm{cms})$ and baseline $b(4 \mathrm{cms})$ of our lag camera. For example, if the object and camera move at the same relative velocity $(R=1)$, the object displaces 14 pixels for a scene distance of $300 \mathrm{cms}$ with image resolution at 1024 by 768 and field of view of 50 degrees.

\section{BACKGROUND SAMPLING}

Since both scene and camera are moving, a lag camera captures surface samples occluded in one camera view with samples captured by another camera (or cameras) from approximately the same viewpoint. This allows us to acquire the background scene even if it is apparently hidden during capture by moving objects. This property is especially useful for dense image acquisition (e.g. lightfields/lumigraphs). It allows us to acquire an environment in less time because we do not have to revisit a location due to occluders temporarily in between camera and scene.

Figure 5a illustrates this property. It depicts a scenario where the object radius $r$ and object velocity $o_{v}$ are such that the object appears completely disjoint in the difference image between corresponded lead camera and follow camera images. In this scenario, the follow camera image of the pair samples a surface occluded in the lead camera image at a previous time instance. Both images are captured from approximately the same viewpoint. However, if the object is too large or moving too slow, the difference image will contain overlapping projections of the moving occluder. The surfaces obscured by the intersection of the two projections of the moving occluder will not be fully sampled by either camera.

From Figure 5a, it is easy to see the projections of the moving object in the difference image will not overlap if the object displacement during the time between lead camera and follow camera images is at least equal to the object diameter, namely $2 r \leq b R$. Given a predetermined object radius $r$, the baseline $b$ for the lag camera, and objects moving amongst the background scene, this expression tells us how fast we can move the lag camera relative to moving objects and still fully capture the background scene despite the occluders. Figure $5 \mathrm{~b}$ graphs the smallest values for $R$ as a function of $r$ for several values of $b$.

\section{IMPLEMENTATION DETAILS}

Our lag camera system uses a pair of Point Grey Research (PGR) Flea cameras rigidly attached to an aluminum base and connected to a standard Dell PC via a Firewire connection. The cameras capture color images of the environment at $1024 \times 768$ pixel resolution and at a rate of $15 \mathrm{~Hz}$. Software for our lag camera is implemented in $\mathrm{C}++$ using standard OpenGL, GLUI, GLUT, and OpenCV libraries. To perform camera calibration, we use an adaptation of Reg Wilson's implementation of the standard Tsai camera model [Tsai 1987]. Calibration indicates the angle between the optical axis of our two cameras is 0.84 degrees and the distance between the two cameras is $4.098 \mathrm{cms}$.

Our prototype lag camera system is attached to a Immersion Corporation MicroScribe G2LX arm [Immersion 2005]. This passive and mechanically tracked arm provides six degrees-of-freedom and a workspace size of 66 inches. Although camera pose can be estimated via a variety of passive methods (e.g., feature tracking, landmark-detection, etc.) or active methods (e.g., magnetic trackers, optical ceiling trackers, inertial sensors, etc.), we use the arm in this implementation so that we can focus on developing lag camera algorithms.

\subsection{MODIFIED UNSTRUCTURED LUMIGRAPH RENDERER}

To demonstrate environment reconstructions, we use a modified unstructured lumigraph renderer (ULR) [Buehler 2001]. A standard ULR system uses the projection of a finely subdivided proxy to determine a 


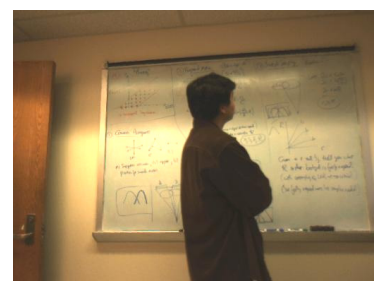

(a)

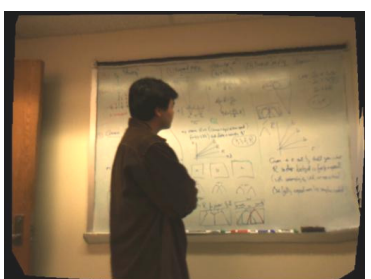

(b)

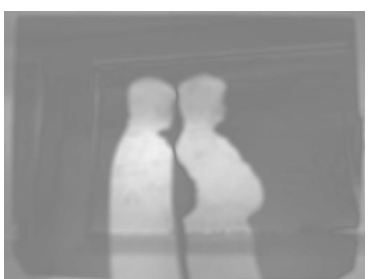

(c)

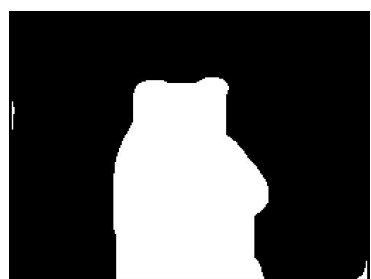

(d)

Figure 6. Processing Pipeline. (a) is the original image from a follow camera, (b) is the warped image of its paired lead camera, (c) is the difference image and (d) is the computed mask. All the images are from the "walk" scene and the followto-lead camera viewpoint distance is $0.351 \mathrm{cms}$.

set of visible surface vertices. A set of weights is computed for each visible surface vertex. These weights determine the set of captured images to use as reference images for generating a novel lumigraph rendering of the scene. We augment the ULR system to also consider the object motion masks computed in Section 4. These masks determine what subset of the reference images can be used to reconstruct the scene. In our implementation, we modify the weighting scheme of a ULR system to include whether the visible surface vertices project onto a valid or invalid region of the reference images. If a vertex falls onto an invalid region, the system will choose the next best reference image until a desired number of reference images are found for each vertex. Our current ULR implementation runs off-line but hardware-friendly implementations have been shown in the past [Buehler 2001] and our method is well suited for these.

\section{RESUlts AND Discussion}

We have constructed and captured several scenes using our prototype 2-view lag camera. Table 1 lists our captured scenes. The five capture sequences have different combinations of scenes, motion, stops, and number of objects. The length of the image sequences range from 102 to 298 images. The video accompanying this paper demonstrates the lag camera, scenes, algorithms, and scene reconstructions.

The lag camera captures, warps, thresholds, creates masks, and display images at interactive rates (about $10 \mathrm{~Hz}$ ). We store in memory $1024 \times 768$ resolution color images from the follow and lead cameras. The differences and masks are generated at quarterresolution $(256 \times 192)$. Image warping and re-projection is done using OpenGL rendering and projective texture-mapping.

Figure 6 demonstrates the sequence of operations for creating a motion mask. Figure $6 a$ shows an example image from a follow camera and Figure $6 \mathrm{~b}$ shows an example warped image from the paired lead camera. The follow camera lags behind the lead camera by 1.125 seconds and comes with 0.351 centimeters of the lead camera viewpoint (average numbers are about the same). Figure 6c shows the absolute value difference image normalized to the range 128 to 255 (e.g., zero difference maps to gray and maximal difference maps to white). Figure $6 \mathrm{~d}$ shows the thresholded binary mask image resulting from the difference image. The proxy, in this case, is a plane registered in place with the observed wall using simple visual approximation.

Figure 7 illustrates how motion masks are reprojected when a moving object temporarily stops. Figures $7(\mathrm{a}, \mathrm{d})$ show the difference and mask images several frames before the motion stops. Figures $7(\mathrm{c}, \mathrm{f})$ show the difference and mask images a few frames after the motion resumes. When the motion stops the difference image is zero (gray). Figure $7 \mathrm{~b}$ shows the difference image one frame before motion stops (so that some of the contour is visible). Nevertheless, we can detect sudden motion change and re-project masks from the surrounding images onto the proxy so as to produce a motion mask for the stop-motion frames.

Figure 8 shows two capture sequences containing moving objects, temporarily stationary objects, and multiple objects. The snapshot of the top sequence is

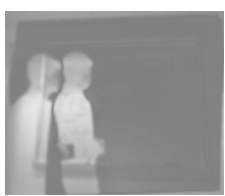

(a)

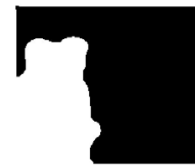

(d)

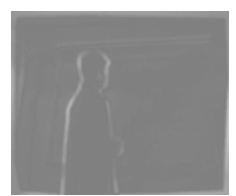

(b)

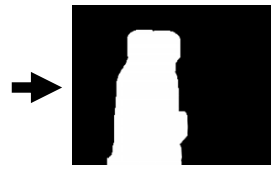

(e)

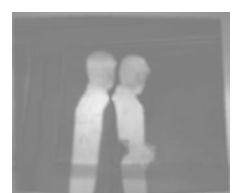

(c)

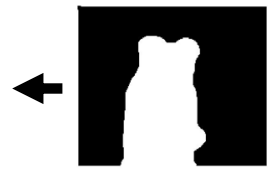

(f)
Figure 7. Stop Motion. (a)(b)(c) are difference images of 3 frames from the "walk-stop-walk" scene. Since motion stops, difference (b) is small. To solve this problem, the mask (d) and (f) are re-projected to the viewpoint of (b) to obtain mask (e). 


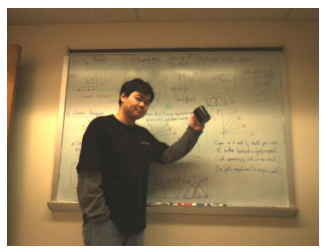

(a)

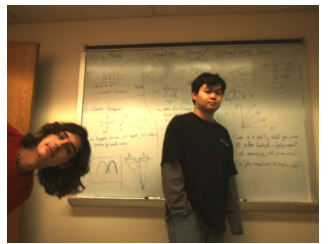

(f)

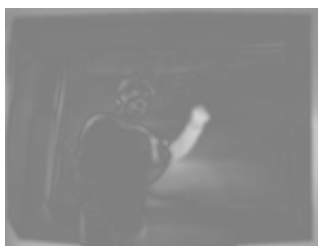

(b)

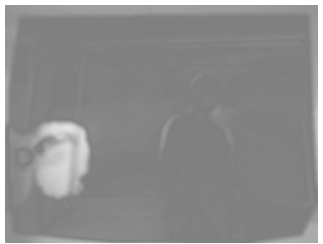

(g)

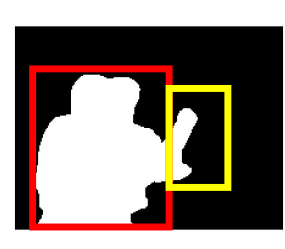

(c)

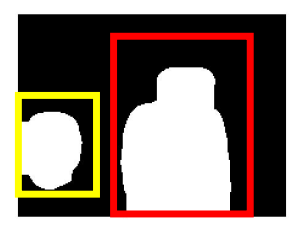

(h)

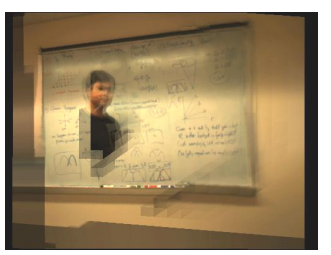

(d)

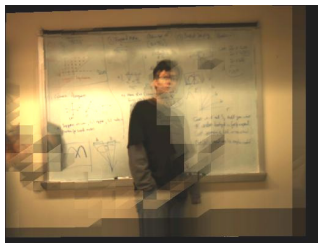

(i)

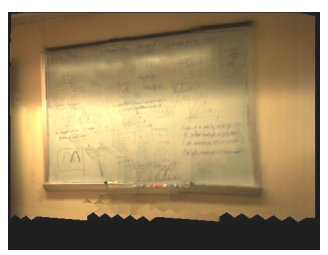

(e)

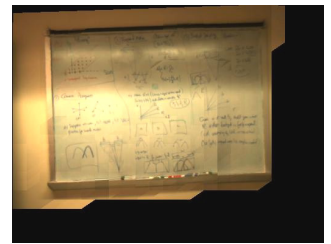

(j)

Figure 8. Multiple Motions. Top row shows the "walk-hi-walk" scene and bottom row shows the "walk-head-walk" scene. (a) and (f) are original frames, (b) and (g) are corresponding difference images (lead camera images not shown). Since, person's body stops, difference (b) is only for the arm motion. Similarly, difference ( $\mathrm{g}$ ) is only for head motion. To obtain motion mask (c) and (f), we re-project neighboring masks to this viewpoint (red rectangle) and then "or" the mask of the arm (head) motion (yellow rectangle). (d, i) and (e, j) are synthesized novel views without and with object detection.

from when the person stopped walking but is moving his arm holding a coffee cup. The snapshot of the bottom sequence is from when the person by the board stops and the person to the left pops his head into the field of view. Figures 8(a, f) show the follow camera images. Figures $8(\mathrm{~b}, \mathrm{~g})$ show difference images from the paired lead camera image (not shown). Figures 8(c, h) demonstrate composite motion masks. In the top sequence the arm motion is an actual motion mask while the stationary person's mask is a re-projection from surrounding frames. Similarly, for the bottom sequence, the person on the left is identified with a regular motion mask and the stationary person's mask is a re-projection. Despite the scene motion, we can properly identify the moving and stationary objects. Figures $8(\mathrm{~d}, \mathrm{i})$ depict a naïve ULR where the images with the moving objects are sometimes selected for rendering. Figures $8(e, j)$ show our improved images using our modified ULR.

Figure 9 contains example images for an office scene. In this example, an intruder attempted to cover and move in front of the scene during capture. Nevertheless, since the lag camera and object are in motion, the space-time sampling allows us to acquire images of the background even from viewpoints where the background surface was temporarily occluded. Figures 9(a-c) demonstrate captured images and Figures 9(d, e) show novel views without and with correct background sampling, respectively.

Our current lag camera implementation only uses image pairs when the distance between follow camera and lead camera is less than a threshold (currently set at $1 \mathrm{~cm}$ ) and the motion is friendly to the intended lag camera design (i.e., the camera motion must be parallel to the camera axis - thus movement parallel to the viewing direction is not supported). Furthermore, if the scene contains occluders that occlude other occluders and they stop and then continue, the handling for stopmotion can be incorrect.

Currently we assume the background is static, the environment is mostly diffuse, and there are no major changes in illumination. Objects in the scene may produce shadows on the background, particularly near the object contour. We do not compensate for these shadows. Furthermore, the Gaussian blurring we perform to smooth the difference images makes object boundaries fuzzy. Thus, to be conservative, our motion masks are slightly dilated to ensure objects are fully contained.

\section{CONCLUSIONS AND FUTURE WORK}

We have introduced the lag camera design and demonstrated an example implementation. A lag camera obtains space-time samples of an environment while the camera is moving through an environment containing moving objects. The camera obtains more than one sample from approximately the same viewpoint but at different and nearby instances in time. This reduces the problem of moving cameras in a moving environment to the case of a static camera in a moving environment. In particular, we demonstrate that a lag camera can easily and interactively detect multiple moving or temporarily stationary objects while itself continuously moving through the environment. Furthermore, the scene behind moving occluders is captured by the lag camera thus enabling 


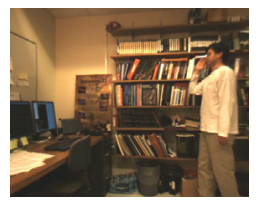

(a)

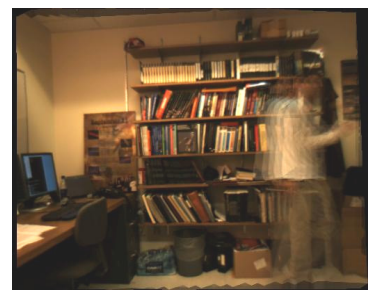

(d)

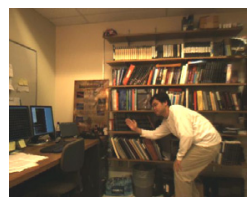

(b)

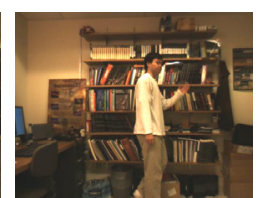

(c)

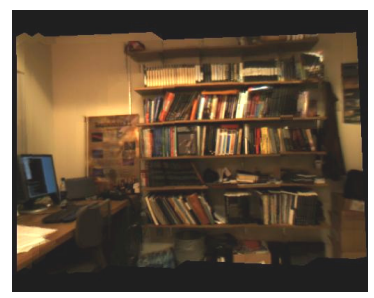

(e)

Figure 9. Background Sampling. We can sample the background of "motion in office" scene even with significant object motion. (a-c) are frames captured and (d-e) are novel views without and with motion masks.

more efficient environment acquisition, even in busy and actively-used environments.

There are several avenues of future work. First, we would like to extend our prototype to use tighter motion masks by "undoubling" the difference images. Second, we wish to add vision-based camera pose estimation to our system to replace mechanically tracked arm. Since the lag camera pairs are from nearby points, we do not expect to have very stringent camera pose estimation requirements. This would enable us to capture large and actively in-use scenes using either a robotic platform carrying a lag-camera or a hand-held/head-mounted lag camera. Third, we are pursuing the explicit acquisition of foreground motion from a moving lag camera.

Finally, since cameras are getting smaller and faster everyday, we are very excited about other lag camera configurations that capture more space-time samples using additional cameras and in a smaller footprint. This enables the acquisition of faster moving scenes. In general, we look forward to future work with lag cameras and believe it could lead to significant new research ideas in acquisition, 3D reconstruction, and space-time processing.

\section{REFERENCES}

[Agarwala 2005] A. Agarwala, C. Zheng, C. Pal, M. Agrawala, M. Cohen, B. Curless, D. Salesin, R. Szeliski. Panoramic Video Textures. ACM Transactions on Graphics (Proceedings of SIGGRAPH 2005), pp. 821-827, 2005.

[Buehler 2001] C. Buehler, M. Bosse, L. McMillan, S. Gortler, and M. Cohen. Unstructured Lumigraph Rendering. Proc. ACM Conference on Computer Graphics (SIGGRAPH), pp. 425-432, 2001.

[Davis 2005] J. Davis, D. Nehab, R. Ramamoorthi, and S. Rusinkiewicz, Spacetime Stereo: A Unifying Framework for Depth from Triangulation, IEEE Transactions on Pattern Analysis and Machine Intelligence, Vol. 27, No. 2, February 2005 .

[Immersion 2005] http://www.emicroscribe.com

[Gortler 1996] S. Gortler, R. Grzeszczuk, R. Szeliski, and M. Cohen. The Lumigraph. Proc. ACM Conference on Computer Graphics (SIGGRAPH), pp. 43-54, 1996.

[Levoy 1996] M. Levoy and P. Hanrahan. Lightfield rendering. Proc. ACM Conference on Computer Graphics (SIGGRAPH), pp. 31--42, 1996.

[Li 2005] Y. Li, J. Sun, and H.-Y. Shum, Video Object Cut and Paste, ACM Trans. on Graphics (Proc. of SIGGRAPH 2005), pp. 595-600, 2005.

[Matusik 2004] W. Matusik and H. Pfister. 3D TV: a Scalable System for Real-time Acquisition, Transmission, and Autostereoscopic Display of Dynamic Scenes. ACM Trans. Graph. (SIGGRAPH Proceeding) 23, 3, pp. 814-824, 2004.

[Naemura 2002] T. Naemura, J. Tago, and H. Harashima. Realtime video-based modeling and rendering of $3 \mathrm{~d}$ scenes. IEEE Computer Graphics and Applications, Vol. 22, No. 2, pp. 66-73, March/April 2002.

[Schödl 2000] A. Schödl, R. Szeliski, D. H. Salesin, and I. Essa. Video textures. Proc. ACM Conference on Computer Graphics (SIGGRAPH), pp. 489-498, July 2000.

[Tsai 1987] R. Tsai, A Versatile Camera Calibration Technique for High-Accuracy 3D Machine Vision Metrology Using Off-the-Shelf TV Cameras and Lenses, IEEE Journal on Robotics and Automation, RA-3:4, pp. 323344, 1987.

[Wang 2005a] H. Wang and R. Yang, Towards Space-Time Light Field Rendering, ACM SIGGRAPH Symposium on Interactive 3D Graphics and Games (I3D), 2005.

[Wang 2005b] J. Wang, P. Bhat, A. Colburn, M. Agrawala and M. Cohen. Interactive Video Cutout. ACM Trans. on Graphics (Proc. of SIGGRAPH), pp. 585-594, 2005.

[Yang 2002] J.C. Yang, M. Everett, C. Buehler, and L. McMillian. A Real-time Distributed Light Field Camera. Proceedings of the 13th Eurographics Workshop on Rendering, pp. 77-86, 2002.

[Zhang 2003] L. Zhang, B. Curless, and S. Seitz, Spacetime Stereo: Shape Recovery for Dynamic Scenes, IEEE Computer Society Conference on Computer Vision and Pattern Recognition (CVPR '03), Volume 2, pp. 367-374, 2003. 